\title{
Comparative effectiveness of sildenafil for pulmonary hypertension due to left heart disease with HFrEF
}

\author{
Rong Jiang ${ }^{1,4}$, Lan Wang ${ }^{1,4}$, Chang-Tai Zhu ${ }^{2}$, Ping Yuan ${ }^{1,4}$, Bigyan Pudasaini ${ }^{3}$, Qin-Hua Zhao ${ }^{1}$, \\ Su-Gang Gong ${ }^{1}$, Jing $\mathrm{He}^{1}$, Jin-Ming $\mathrm{Liu}^{1}$ and Qing-Hua $\mathrm{Hu}^{1}$
}

There is no cure for pulmonary hypertension due to left heart disease (PH-LHD), but the rationale for using sildenafil to treat pulmonary arterial hypertension with heart failure with reduced ejection fraction (HFrEF) has been supported by short-term studies. We performed a meta-analysis to evaluate the effectiveness of sildenafil for PH-LHD with HFrEF. A systematic literature search of PubMed, EMBASE and the Cochrane Central Register of Controlled Trials was conducted from inception through October $\mathbf{2 0 1 4}$ for randomized trials and for observational studies with control groups, evaluating the effectiveness of sildenafil to treat PH-LHD with HFrEF. Sildenafil therapy decreased pulmonary arterial systolic pressure both at the acute phase and at the 6-month follow-up (weighted mean difference (WMD): $-6.03 \mathrm{~mm} \mathrm{Hg}, P=0.02$; WMD: $-11.47 \mathrm{~mm} \mathrm{Hg}, P<0.00001$, respectively). Sildenafil was found to reduce mean pulmonary artery pressure (WMD: $-3 \mathrm{~mm} \mathrm{Hg}, P=0.0004$ ) and pulmonary vascular resistance (WMD: -60.0 dynes $\mathrm{cm}^{-5}, P=0.01$ ) at the 3-month follow-up. Oxygen consumption at peak significantly increased to $3.66 \mathrm{ml} \mathrm{min}{ }^{-1} \mathrm{~kg}^{-1}(P<0.00001), 3.36 \mathrm{ml} \mathrm{min}^{-1} \mathrm{~kg}^{-1}(P<0.00001)$ and $2.60 \mathrm{ml} \mathrm{min}{ }^{-1} \mathrm{~kg}^{-1}(P=0.03)$ at 3 , 6 and 12 months, respectively. There were significant reductions in ventilation to $\mathrm{CO}_{2}$ production slope of $-2.00,-4.68$ and -7.12 at 3,6 and 12 months, respectively $(P<0.00001)$. Sildenafil was superior to placebo regarding left ventricular ejection fraction at the 6-month follow-up (WMD: 4.35, $P<0.00001$ ), and it significantly improved quality of life. Sildenafil therapy could effectively improve pulmonary hemodynamics and cardiopulmonary exercise testing measurements of PH-LHD with HFrEF, regardless of acute or chronic treatment.

Hypertension Research (2015) 38, 829-839; doi:10.1038/hr.2015.73; published online 23 July 2015

Keywords: meta-analysis; phosphodiesterase type- 5 inhibitors; pulmonary hypertension; randomized controlled trials

\section{INTRODUCTION}

Pulmonary hypertension $(\mathrm{PH})$ is a common complication of left heart disease (LHD), frequently occurring as a 'symptom' of the underlying condition. $^{1,2} \mathrm{PH}$ associated with LHD is classified as group II PH (postcapillary $\mathrm{PH})^{3,4}$ The current hemodynamic definition of PH-LHD combines a mean pulmonary artery pressure (mPAP) $>25 \mathrm{~mm} \mathrm{Hg}$, a pulmonary artery wedge pressure $>15 \mathrm{~mm} \mathrm{Hg}$ and a normal or reduced cardiac output (CO). ${ }^{3,4}$ Irrespective of the origin of LHD, the first event leading to $\mathrm{PH}$ (isolated postcapillary $\mathrm{PH})^{4}$ is passive backward transmission of filling pressures, mainly driven by left ventricular (LV) diastolic dysfunction. ${ }^{5}$ These purely mechanical components of venous congestion trigger a superimposed component, combining pulmonary vasoconstriction, decreased nitric oxide (NO) availability, ${ }^{6}$ increased endothelin expression, desensitization to natriuretic peptide-induced vasodilation and vascular remodeling., ${ }^{2,7}$ At this stage, mPAP increases further, and this increase seems to occur in excess of elevated pulmonary artery wedge pressure. ${ }^{5}$ Finally, these changes lead to pulmonary vascular disease (combined postcapillary and precapillary $\mathrm{PH}),{ }^{4}$ increased right ventricle (RV) afterload and RV failure.

$\mathrm{PH}$ in LHD is most common in patients with heart failure (HF), with preserved or reduced ejection fraction (EF). ${ }^{1,2,8} \mathrm{PH}-\mathrm{LHD}$ results in more severe symptoms. It causes worse exercise intolerance, exerts a negative impact on outcomes and is often related to disease severity. HF with preserved ejection fraction (HFpEF) is a common cause of $\mathrm{PH}$, associated with worse outcomes than those with HF with reduced ejection fraction (HFrEF). ${ }^{2,3,9,10}$ A multicenter, double-blind, placebocontrolled, parallel study (RELAX) conducted by the Heart Failure Clinical Research Network ${ }^{11}$ failed to prove that taking sildenafil for 24 weeks could alter exercise capacity or clinical status compared with placebo in patients with HFpEF. Compared with HFpEF, PH-LHD with HFrEF was shown to improve with additional sildenafil therapy, as shown by some single-center, double-blind, placebo-controlled randomized controlled trials (RCTs). ${ }^{12-20}$

\footnotetext{
${ }^{1}$ Department of Cardio-Pulmonary Circulation, Shanghai Pulmonary Hospital, Tongji University School of Medicine, Shanghai, China; ${ }^{2}$ Department of Transfusion Medicine, Shanghai Jiao Tong University Affiliated Sixth People's Hospital, Shanghai, China and ${ }^{3}$ Tongji University School of Medicine, Shanghai, China ${ }^{4}$ These authors contributed equally to this work.

Correspondence: Professor J-M Liu or Professor Q-H Hu, Department of Cardio-Pulmonary Circulation, Shanghai Pulmonary Hospital, Tongji University School of Medicine, Shanghai 200433, China. 
Compared with pulmonary arterial hypertension (PAH) patients, patients with PH-LHD are more often heterogeneous older women with a history of systemic hypertension, ${ }^{21}$ having most if not all of the features of metabolic syndrome $e^{22}$ and extensive medical histories resulting in complex polypharmacy. There are no validated treatments for $\mathrm{PH}-\mathrm{LHD}$, particularly HFpEF. The rationale for using $\mathrm{PAH}$ therapies in PH-LHD with HF and HFrEF has been supported by acute or short-term studies using phosphodiesterase type-5 (PDE5) inhibitors (sildenafil).

PDE5 is the principal enzyme responsible for cyclic guanosine monophosphate (cGMP) catabolism in the lungs, and it has been shown in animal models to be upregulated in pulmonary vascular smooth muscle cells under conditions associated with $\mathrm{PH} .{ }^{23}$ The beneficial effect of sildenafil on pulmonary hemodynamic parameters arises from selective inhibition of isoform 5 (a cGMP-specific PDE) in the pulmonary vasculature. ${ }^{24,25}$

Most of these studies have consistently reported that there have been improvements in hemodynamic status, exercise capacity and symptoms. However, the methodologies (small sample sizes, single centers, unclear or no randomization processes) have not provided sufficient evidence to support the use of these drugs in the clinical management of patients.

A meta-analysis by Huang et al. ${ }^{26}$ including six RCTs concluded that additional sildenafil treatment was a potential therapeutic method to improve pulmonary exercise capacity and quality of life (QoL) in patients with chronic systolic HF. However, the meta-analysis did not include three RCTs ${ }^{16,18,19}$ and one observational study ${ }^{27}$ with a control group. It also did not compare the effects of short- and long-term therapy separately.

We sought to improve upon the previous meta-analysis by addressing these issues. By pooling the available literature, we sought to assess the impact of sildenafil for PH-LHD with HFrEF, including hemodynamics, exercise tolerance and QoL, by comparing the different phases separately.

\section{MATERIALS AND METHODS}

\section{Data sources and search strategy}

This study was designed according to the PRISMA statement (preferred reporting items for systematic reviews and meta-analyses). ${ }^{28}$ We searched PubMed, EMBASE and the Cochrane Collaboration databases using the key words 'pulmonary hypertension', 'heart failure', 'sildenafil' and 'phosphodiesterase type- 5 inhibitors' to identify RCTs and observational studies with a control group that evaluated the effects of sildenafil for PH-LHD. Our search included articles published from database inception until October 2014. The search was limited to English language articles. In addition, we manually searched references of retrieved articles, and we used PubMed's 'related articles' feature to identify studies not captured by our primary search design.

\section{Study selection}

We included nine RCTs and one observational trial with controls comparing the effectiveness response in PH-LHD patients treated with sildenafil versus placebo. The inclusion criteria were as follows: (1) receiving drug treatment and in a clinically stable condition (defined as no changes in HF regimens or hospitalization prior to study entry); (2) HFrEF; (3) included PH-LHD assessed by right heart catheterization or echocardiography; (4) negative exercise stress test; (5) no history of sildenafil intolerance, significant lung or valvular heart diseases, severe arrhythmia, neuromuscular disorders or peripheral vascular disease; (6) not taking long-acting nitrate preparations; (7) not receiving sildenafil at the time of study; and (8) no other pharmacotherapy with endothelin antagonists (bosentan, darusentan), prostanoids (epoprostenol, treprostinil, iloprost) or other PDE5 inhibitors (tadalafil). Studies were excluded if they were self-control studies. Reviews, editorials, letters, animal studies, case reports and conference abstracts were also excluded.

\section{Data extraction}

The literature search, data extraction and methodological grading were performed in duplicate by two independent reviewers (RJ, PY). Disagreements were resolved by consensus. We extracted data pertaining to the characteristics of all of the studies (number of subjects, type of trial, inclusion/exclusion criteria, length of follow-up, primary end points, results). The pooled efficacy outcomes noted were pulmonary hemodynamic measurements, cardiopulmonary exercise testing (CPET) variables, systolic hemodynamic measures, left ventricle ejection fraction (LVEF) and QoL.

\section{Outcomes}

The primary outcome measurements were improvements in hemodynamic parameters and CPET after receiving sildenafil therapy versus placebo during the acute phase and over long-term follow-up. Secondary outcome measurements included improvements in LVEF and QoL between the acute phase and long-term follow-up.

\section{Methodological quality}

The risk of bias was assessed using domains suggested by the Cochrane Handbook of Systematic Reviews, specifically emphasizing sequence generation, allocation concealment, blinding, outcomes assessment and selective reporting for the nine RCTs (Supplementary Figure S1). ${ }^{29}$ We used the NewcastleOttawa scale to assess the risk of bias for the observational studies (Supplementary Table S1).$^{30}$ Publication bias was assessed using Begg's adjusted rank correlation test (Supplementary Figure S2).

\section{Data synthesis and statistical analysis}

We conducted meta-analyses for comparisons when two or more studies reported the same outcome. Continuous variables were analyzed using weighted mean difference (WMD) or standardized mean differences. Throughout the article, values are presented as the mean \pm s.ds. unless otherwise stated. We used random-effect models or fixed-effect models to synthesize quantitatively the available evidence and to calculate summary estimates. For controlled studies, the difference in outcome measurement changes with sildenafil versus placebo was pooled across studies and was analyzed using a random-effect or fixed-effect model to compare heterogeneity using the $I^{2}$ statistic. Pooled analysis was calculated using fixed-effect models, and random-effect models were applied in cases of significant heterogeneity across studies. Separate models were constructed for the acute phase and at 3,6 or 12 months of follow-up.

In our initial analysis, certain studies reported measurements of variability other than s.d. The only measurement of variability reported was the interquartile range. These cases, in which $95 \%$ confidence intervals or s.e.m. failed to convert to s.d., were excluded to maintain the consistency of the reported results. By including this study in the meta-analysis models, sensitivity analyses were performed. We considered $P<0.05$ as significant.

The analysis was performed using the Stata software, version 12 (Stata, College Station, TX, USA), and RevMan software, version 5.1 (The Cochrane Collaboration, Copenhagen, Denmark).

\section{RESULTS}

\section{Characteristics of the studies}

Of the 217 articles identified by the initial search, 112 articles were retrieved for more detailed evaluation, and 8 trials were included in the study (Figure 1). Ten studies were included in our systematic review, encompassing 299 patients treated between 2000 and 2013. The characteristics of all of the included studies are presented in Table 1. There were nine $\mathrm{RCTs}^{12-20}(n=286)$ and one observational trial $^{27}$ with a control group $(n=13)$.

\section{Pulmonary hemodynamic measurements}

In five RCTs, ${ }^{14,16-18,20}$ sildenafil therapy decreased pulmonary artery systolic pressure (PASP) by $-6.03 \mathrm{~mm} \mathrm{Hg}(95 \% \mathrm{CI}:-11.10$ to $-0.97 \mathrm{~mm} \mathrm{Hg}, P=0.02)$ and $-11.47 \mathrm{~mm} \mathrm{Hg}(95 \% \mathrm{CI}:-14.70$ to 


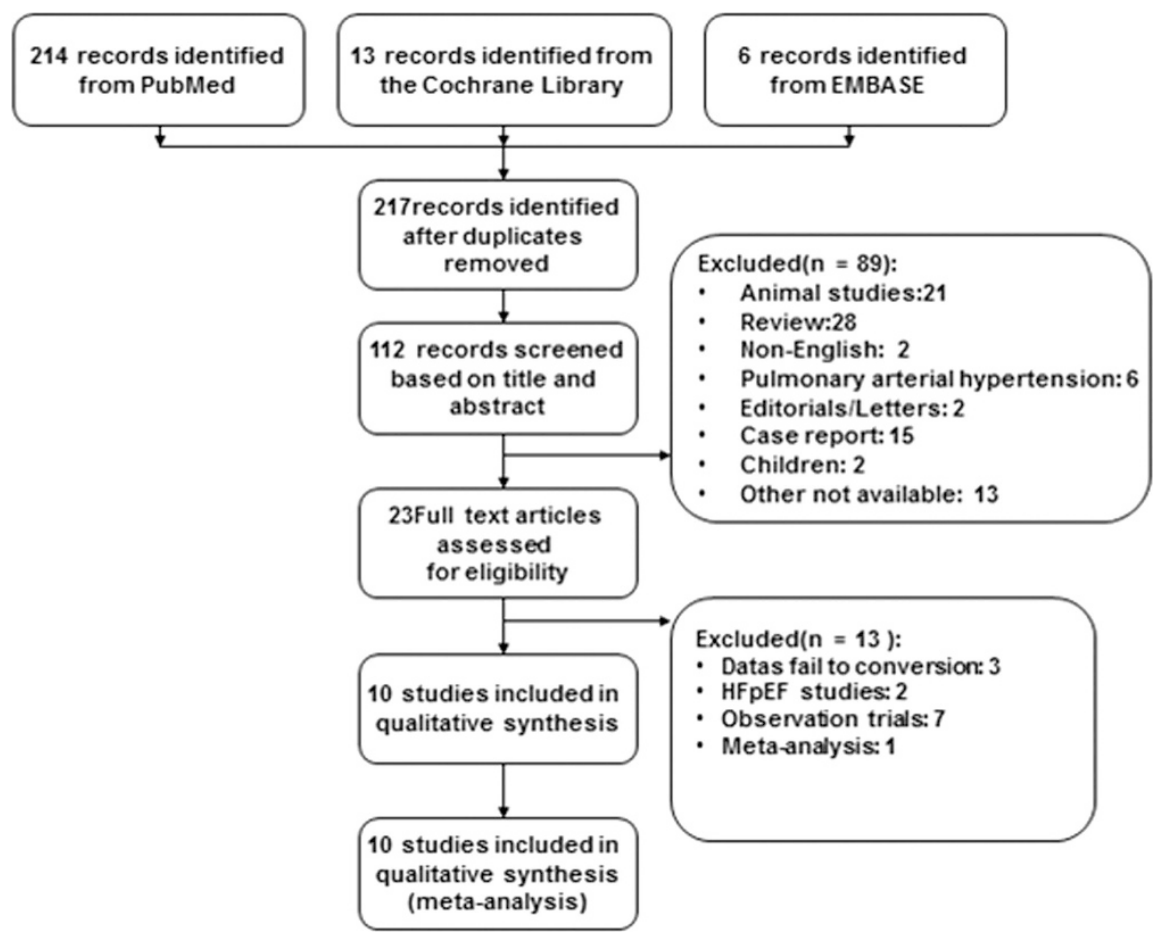

Figure 1 Search flow diagram for studies included in the meta-analysis.

$-8.24 \mathrm{~mm} \mathrm{Hg}, P<0.00001)$ at the acute phase and at the 6-month follow-up, respectively, in $\mathrm{PH}$ patients secondary to HFrEF, with significant heterogeneity between the studies $\left(I^{2}=96 \%\right.$ and $84 \%$, respectively). Compared with placebo, sildenafil therapy was found to reduce $\mathrm{mPAP}$ (WMD: $-3 \mathrm{~mm} \mathrm{Hg}, 95 \% \mathrm{CI}:-4.66$ to $-1.34 \mathrm{~mm} \mathrm{Hg}$, $P=0.0004$ ), and pulmonary vascular resistance (PVR) (WMD: -60.0 dynes $\mathrm{cm}^{-5}, \quad 95 \%$ CI: -105.51 to -14.49 dynes $\mathrm{cm}^{-5}$, $P=0.010)$ at the 3-month follow-up. However, the pulmonary capillary wedge pressure (PCWP) remained unchanged (WMD: $-1.0 \mathrm{~mm} \mathrm{Hg}, P=0.13$ ) at the 3-month follow-up. No heterogeneity was observed in mPAP, PVR or PCWP (for all, $I^{2}=0$; Figures $2 \mathrm{a}-\mathrm{d}$ ).

\section{Systematic hemodynamic measurements}

In six of the included RCTs, ${ }^{12,13,16-19}$ sildenafil therapy did not influence resting systolic blood pressure (SBP), diastolic blood pressure $(\mathrm{DBP})$ or heart rate $(\mathrm{HR})$ regardless of acute phase or long-term follow-up.

Although sildenafil therapy increased HR (WMD: 2.89 beats per minute (b.p.m.), 95\% CI: 0.85 to 4.93 b.p.m.) with no heterogeneity $\left(I^{2}=0\right)$ at 3 months of follow-up. However, there were changes in HR of 0.63 b.p.m. (95\% CI: -1.85 to 3.11 b.p.m., $P=0.62$ ), 0.19 b.p.m. ( $95 \%$ CI: -3.13 to 3.52 b.p.m., $P=0.91$ ) and 0.96 b.p.m. (95\% CI: -0.64 to 2.56 b.p.m., $P=0.24$ ) at the acute phase, the 6 -month follow-up and overall, respectively (Figure $3 \mathrm{a}$ ).

There were changes in SBP and DBP during the acute phase of $-1.46 \mathrm{~mm} \mathrm{Hg}$ (95\% CI: -4.25 to $1.33 \mathrm{~mm} \mathrm{Hg}$ ) and $-0.78 \mathrm{~mm} \mathrm{Hg}$ ( $95 \% \mathrm{CI}:-2.15$ to $0.58 \mathrm{~mm} \mathrm{Hg}$ ) with sildenafil therapy versus placebo, respectively (for both $P>0.05$ ). At 6 months, there were also reductions of $1.94 \mathrm{~mm} \mathrm{Hg}$ in SBP (95\% CI: -0.75 to $4.63 \mathrm{~mm} \mathrm{Hg}$ ) and $0.78 \mathrm{~mm} \mathrm{Hg}$ (95\% CI: -1.11 to $3.66 \mathrm{~mm} \mathrm{Hg}$ ), respectively. There was a modest amount of heterogeneity (all $I^{2}=0$ except $I^{2}=47 \%$ during the acute phase for SBP; Figures $3 \mathrm{~b}$ and $\mathrm{c}$ ).

\section{Cardiopulmonary exercise testing}

The patients performed a progressively increasing work rate CPET to maximal tolerance on an electromagnetically braked cycle ergometer in the upright position. The oxygen consumption at peak exercise (peak $\mathrm{VO}_{2}$ ), the efficiency of ventilatory response to carbon dioxide output ( $\mathrm{VE} / \mathrm{VCO}_{2}$ slope), oxygen consumption at the anaerobic threshold (AT) $\left(\mathrm{VO}_{2}\right.$ at $\left.\mathrm{AT}\right)$, the ratio of dead space to tidal volume at peak exercise (peak VD/VT), peak respiratory exchange ratio (RER), peak workload and aerobic efficiency (the ratio of change in oxygen uptake to minute ventilation) $\left(\Delta \mathrm{VO}_{2} / \Delta \mathrm{WR}\right)$ were evaluated to determine cardiopulmonary exercise capacity.

With regard to CPET, six RCTs ${ }^{13,14,16-19}$ and one observational study ${ }^{27}$ were included to analyze peak $\mathrm{VO}_{2}$ and $\mathrm{VE} / \mathrm{VCO}_{2}$ slope.

There was no significant increase in peak $\mathrm{VO}_{2}$ at acute duration $\left(0.84 \mathrm{ml} \mathrm{min}^{-1} \mathrm{~kg}^{-1}, \quad 95 \% \quad \mathrm{CI}:-0.38\right.$ to $2.07 \mathrm{ml} \mathrm{min}^{-1} \mathrm{~kg}^{-1}$; $\left.P=0.18, I^{2}=80 \%\right)$. However, there were significant increases in peak $\mathrm{VO}_{2}$ of $3.66 \mathrm{ml} \mathrm{min}{ }^{-1} \mathrm{~kg}^{-1}$ (95\% CI: 2.74 to $4.57 \mathrm{ml} \mathrm{min}^{-1} \mathrm{~kg}^{-1}$, $\left.P<0.00001, \quad I^{2}=0 \%\right), \quad 3.36 \mathrm{ml} \mathrm{min}^{-1} \mathrm{~kg}^{-1} \quad(95 \% \quad \mathrm{CI}: 2.46$ to $\left.4.27 \mathrm{ml} \mathrm{min}^{-1} \mathrm{~kg}^{-1}, P<0.00001, I^{2}=0 \%\right)$ and $2.60 \mathrm{ml} \mathrm{min}^{-1} \mathrm{~kg}^{-1}$ ( $95 \%$ CI: 0.23 to $4.97 \mathrm{ml} \mathrm{min}^{-1} \mathrm{~kg}^{-1}, P=0.03, I^{2}=0 \%$ ) at 3,6 and 12 months, respectively, with sildenafil. Compared with placebo, there was an increase in peak $\mathrm{VO}_{2}$ of $1.97 \mathrm{ml} \mathrm{min}{ }^{-1} \mathrm{~kg}^{-1}$ (95\% CI: 0.76 to $3.17 \mathrm{ml} \mathrm{min}^{-1} \mathrm{~kg}^{-1}, P=0.002, I^{2}=79.4 \%$ ) overall (Figure 4a).

There was no significant reduction in $\mathrm{VE} / \mathrm{VCO}_{2}$ slope at the acute duration of $-0.31(95 \% \mathrm{CI}:-2.89$ to $2.27 ; P=0.81)$. There were significant reductions in the $\mathrm{VE} / \mathrm{VCO}_{2}$ slope of $-2.00(95 \% \mathrm{CI}$ : -3.74 to $\left.-0.26, P<0.00001, I^{2}=0 \%\right),-4.68$ (95\% CI: -5.95 to $\left.-3.41, P<0.00001, I^{2}=0 \%\right)$ and -7.12 (95\% CI: -8.83 to -5.41 , $\left.P<0.00001, I^{2}=0 \%\right)$ at 3,6 and 12 months, respectively. Compared with placebo, there was a reduction in $\mathrm{VE} / \mathrm{VCO}_{2}$ slope of -2.70 (95\% CI: -4.66 to -0.75$)$ overall $\left(P=0.007, I^{2}=87 \%\right)$. Compared with significant heterogeneity at the acute phase $\left(I^{2}=81 \%\right)$, there was no heterogeneity at 3,6 or 12 months $\left(I^{2}=0 \%\right.$; Figure $\left.4 \mathrm{~b}\right)$. 
Table 1 Characteristics and inclusion criteria of all included studies

\begin{tabular}{|c|c|c|c|c|c|c|}
\hline $\begin{array}{l}\text { Drug/ } \\
\text { author }\end{array}$ & $\begin{array}{l}\text { No. of } \\
\text { patients }\end{array}$ & $\begin{array}{l}\text { Design (type of } \\
\text { the study) }\end{array}$ & Inclusion/exclusion criteria & $\begin{array}{l}\text { Length of } \\
\text { follow-up }\end{array}$ & Primary end point & Results \\
\hline $\begin{array}{l}\text { Bocchi } \\
\text { et al. }{ }^{19}\end{array}$ & $\begin{array}{l}n=24 \\
\text { NYHA } \\
\text { II-IV }\end{array}$ & $\begin{array}{l}\text { Nearly } 1: 1 \\
\text { RCT dose of } \\
50 \mathrm{mg}\end{array}$ & $\begin{array}{l}\text { Standard criteria }{ }^{a} \text { no exception to } \mathrm{HR}<55 \text { b.p.m., high-risk } \\
\text { arrhythmias, } \mathrm{SBP}<85 \mathrm{~mm} \mathrm{Hg} \text {, low systemic perfusion, } \\
\text { venous or pulmonary congestion; no female }\end{array}$ & $\begin{array}{l}\text { Acute; } \\
4 \text { weeks }\end{array}$ & $\begin{array}{l}\text { CPET variables; HR, } \\
\text { SBP, DBP }\end{array}$ & $\begin{array}{l}\mathrm{HR}, \mathrm{SBP}, \mathrm{DBP} \text { and } \mathrm{VE} / \mathrm{VCO}_{2} \downarrow \\
\text { peak } \mathrm{VO}_{2} \uparrow\end{array}$ \\
\hline $\begin{array}{l}\text { Guazzi } \\
\text { et al. }{ }^{16}\end{array}$ & $\begin{array}{l}n=24 \\
\mathrm{NYHA} \\
\|-\| I\end{array}$ & $\begin{array}{l}2: 1 \mathrm{RCT} \text { dose } \\
\text { of } 50 \mathrm{mg}\end{array}$ & $\begin{array}{l}\text { Standard criteria }{ }^{a} \text { but no exception to hypertension, } \\
\mathrm{CoHb}>2 \% \text {, no female }\end{array}$ & Acute & $\begin{array}{l}\text { Hemodynamics; } \\
\text { CPET; pulmonary } \\
\text { function test }\end{array}$ & $\begin{array}{l}\mathrm{DLco} \text {, exercise peak } \mathrm{VO}_{2} \\
\mathrm{VE} / \mathrm{VCO}_{2} \text { slope aerobic } \\
\left(\Delta \mathrm{VO}_{2} / \Delta \mathrm{WR}\right) \uparrow\end{array}$ \\
\hline $\begin{array}{l}\text { Semigran, } \\
\text { et al. }{ }^{12}\end{array}$ & $\begin{array}{l}n=34 \\
\text { NYHA } \\
\text { II-IV }\end{array}$ & $\begin{array}{l}1: 1 \mathrm{RCT} \text { dose } \\
\text { of } 25-50 \mathrm{mg} \\
\text { tid }\end{array}$ & Standard criteria ${ }^{\mathrm{a}}$ with $\mathrm{PH}$ & 12 weeks & $\begin{array}{l}\text { CPET variables; } \\
\text { hemodynamics; QoL }\end{array}$ & $\begin{array}{l}\text { Peak } \mathrm{VO}_{2}, \mathrm{CO}, 6 \mathrm{MWT} \uparrow . \text { No } \\
\text { change in others }\end{array}$ \\
\hline $\begin{array}{l}\text { Semigran, } \\
\text { et al. }{ }^{27}\end{array}$ & $\begin{array}{l}n=13 \\
\text { NYHA } \\
\text { III }\end{array}$ & Dose of $50 \mathrm{mg}$ & Standard criteria ${ }^{a}$ with $\operatorname{LVEF}<35 \%$ & Acute & $\begin{array}{l}\text { Exercise capacity; } \\
\text { exercise } \\
\text { hemodynamics }\end{array}$ & $\begin{array}{l}\text { Decreased resting and exercise } \\
\text { PAP, PVR, VE/ } \mathrm{VCO}_{2} \text { slope; } \\
\text { Increased resting and exercise } \mathrm{CI} \text {, } \\
\text { peak } \mathrm{VO}_{2}\end{array}$ \\
\hline $\begin{array}{l}\text { Clausell, } \\
\text { et al. }\end{array}$ & $\begin{array}{l}n=19 \\
\text { NYHA } \\
\text { I-III }\end{array}$ & $\begin{array}{l}1: 1 \mathrm{RCT} \text { dose } \\
\text { of } 50 \mathrm{mg} \text { tid }\end{array}$ & $\begin{array}{l}\text { Standard criteria }{ }^{a} \text { with clinically stable } \geqslant 2 \text { months, except } \\
\mathrm{HR}<55 \text { b.p.m., SBP }<90 \mathrm{~mm} \mathrm{Hg} \text {, arrial fibrillation, oral } \\
\text { anticoagulation }\end{array}$ & $\begin{array}{l}\text { Acute, } \\
\text { 4-week } \\
\text { duration }\end{array}$ & $\begin{array}{l}\text { CPET variables; } \\
\text { hemodynamics }\end{array}$ & $\begin{array}{l}\text { Increased peak } \mathrm{VO}_{2} \text {, SPAP, } \\
\mathrm{VE} / \mathrm{VCO}_{2} \text { slope. No change in } \\
\text { others }\end{array}$ \\
\hline $\begin{array}{l}\text { Lewis } \\
\text { et al. }{ }^{13}\end{array}$ & $\begin{array}{l}n=30 \\
\text { NYHA } \\
\text { II-IV }\end{array}$ & $\begin{array}{l}1: 1 \mathrm{RCT} \text { dose } \\
\text { of } 25-75 \mathrm{mg} \\
\text { tid }\end{array}$ & Standard criteria ${ }^{\mathrm{a}}$ with $\mathrm{PH}$ & 12 weeks & $\begin{array}{l}\text { CPET variables; } \\
\text { hemodynamics }\end{array}$ & $\begin{array}{l}\text { VE/VCO } \\
\text { associated with PVR, PCWP }\end{array}$ \\
\hline $\begin{array}{l}\text { Guazzi } \\
\text { et al. }{ }^{18}\end{array}$ & $\begin{array}{l}n=32 \\
\text { NYHA } \\
\|-I I I\end{array}$ & $\begin{array}{l}1: 1 \text { randomi- } \\
\text { zation dose of } \\
50 \mathrm{mg}\end{array}$ & Chronic heart failure no exception to female & Acute & FMD; CPX variables & $\begin{array}{l}\text { Decreased } \mathrm{VE} / \mathrm{VCO}_{2} \text { slope, } \\
\text { increased } \Delta \mathrm{VO}_{2} / \mathrm{WR}, \mathrm{PAP} \text {, } \\
\text { peak } \mathrm{VO}_{2}, \mathrm{LVEF}\end{array}$ \\
\hline $\begin{array}{l}\text { Guazzi } \\
\text { et al. }{ }^{17}\end{array}$ & $\begin{array}{l}n=46 \\
\text { NYHA } \\
\text { II-III }\end{array}$ & $\begin{array}{l}1: 1 \mathrm{RCT} \text { dose } \\
\text { of } 50 \mathrm{mg} \text { tid }\end{array}$ & $\begin{array}{l}\text { Standard criteria }{ }^{a} \text { no exception to } \mathrm{SBP}>140 \text { or }<110 \mathrm{~mm} \\
\mathrm{Hg} \text {, diabetes mellitus, atrial fibrillation, no female }\end{array}$ & $\begin{array}{l}\text { Acute; } \\
3 \text { and } \\
6 \text { months }\end{array}$ & $\begin{array}{l}\text { CPET variables; } \\
\text { hemodynamics; FMD }\end{array}$ & $\begin{array}{l}\text { SPAP } \downarrow \text {; peak } \mathrm{VO}_{2}, \text { VE } / \mathrm{VCO}_{2} \text { slope, } \\
\text { QoL } \uparrow\end{array}$ \\
\hline $\begin{array}{l}\text { Guazzi } \\
\text { et al. }{ }^{11,14}\end{array}$ & $\begin{array}{l}n=45 \\
\text { NYHA } \\
\|-\| I\end{array}$ & $\begin{array}{l}2: 1 \mathrm{RCT} \text { dose } \\
\text { of } 50 \mathrm{mg} \text { tid }\end{array}$ & $\begin{array}{l}\text { Standard criteria }{ }^{a} \text { no exception to SBP }<110 \mathrm{~mm} \mathrm{Hg} \text {, LV } \\
\text { assist devices. }\end{array}$ & $\begin{array}{l}\text { Acute; } \\
6 \text { months } \\
1 \text { year }\end{array}$ & $\begin{array}{l}\text { Hemodynamics; } \\
\text { echocardiography; } \\
\text { CPET; QoL; hormones }\end{array}$ & $\begin{array}{l}\text { CPET variables } \uparrow, \text { QoL } \uparrow \text {, hor- } \\
\text { mones } \uparrow \text {; no change in others }\end{array}$ \\
\hline $\begin{array}{l}\text { Guazzi } \\
\text { et al. }{ }^{15}\end{array}$ & $\begin{array}{l}n=32 \\
\text { NYHA } \\
\text { III-IV }\end{array}$ & $\begin{array}{l}1: 1 \mathrm{RCT} \text { doses } \\
\text { of } 50 \mathrm{mg} \text { tid }\end{array}$ & $\begin{array}{l}\text { Standard criteria }{ }^{a} \text { with } \mathrm{PH} \text { but no exception to SBP }>140 \text { or } \\
<110 \mathrm{~mm} \mathrm{Hg} \text {; no female }\end{array}$ & $\begin{array}{l}6 \text { months, } \\
1 \text { year }\end{array}$ & $\begin{array}{l}\text { Hemodynamics; } \\
\text { CPET variables; QoL }\end{array}$ & $\begin{array}{l}\text { Peak } \mathrm{VO}_{2} \uparrow, \mathrm{VE} / \mathrm{VCO}_{2} \text { slope } \uparrow \\
\text { PCWP } \downarrow, \mathrm{mPAP} \downarrow, \mathrm{PVR} \downarrow ; \mathrm{QoL} \uparrow\end{array}$ \\
\hline
\end{tabular}

Abbreviations: BP, blood pressure; b.p.m., beats per minute; CHD, chronic heart disease; Cl, cardiac index; CO, cardiac output; CoHb, carboxyhemoglobin; CPET, cardiopulmonary exercise testing; CPX, Cardiopulmonary Exercise Testing; DBP, diastolic blood pressure; DLco, lung diffusion for carbon monoxide; ED, erectile dysfunction; FEV1/FVC, forced expiratory volume 1 s/forced vital capacity ratio; FMD, flow-mediated brachial artery dilation; HF, heart failure; HR, heart rate; LV, left ventricular; LVAD, LV assist device; LVEDP, LV end-diastolic pressure; LVEF, LV ejection fraction; LVESP, LV end-systolic pressure; MAP, mean arterial pressure; mPAP, mean pulmonary arterial pressure; MRI, magnetic resonance imaging; NO, nitric oxygen; NYHA, New York Heart Association functional class; PAWP, pulmonary artery wedge pressure; PCWP, pulmonary capillary wedge pressure; PH, pulmonary hypertension; PVR, pulmonary vascular resistance; QoL, quality of life; RCT, randomized controlled trial; SBP, systolic blood pressure; SPAP, systolic pulmonary arterial pressure; SVR, systemic vascular resistance; TPG, transpulmonary gradient; VE/VCO 2 slope, slope of increase in ventilation versus carbon dioxide diffusion for carbon output; $\mathrm{VO}_{2}$, oxygen consumption; $6 \mathrm{MWT}, 6$-min walking distances; $\Delta \mathrm{VO}_{2} / \Delta \mathrm{WR}$, the ratio of change in oxygen uptake versus minute ventilation.

versus minute ventilation. 40 or $45 \%$ (required by different studies); no treatment with long-acting nitrate preparations; no history of intolerance of sildenafil and myocardial infarction, unstable angina, stroke, lung diseases with FEV1/FVC $>70 \%$, valvular diseases, neuromuscular diseases; and not receiving sildenafil at the time of study.

There was a significant increase in $\mathrm{VO}_{2}$ at an AT of $0.90 \mathrm{ml} \mathrm{min}^{-1} \mathrm{~kg}^{-1}$ (95\% CI: -0.29 to $2.08 \mathrm{ml} \mathrm{min}^{-1} \mathrm{~kg}^{-1}$ ) at the acute duration $\left(I^{2}=0 \%\right)$. The values of $\mathrm{VO}_{2}$ at the AT increase were $2.61 \mathrm{ml} \mathrm{min}^{-1} \mathrm{~kg}^{-1}$ (95\% CI: 0.86 to $4.36 \mathrm{ml} \mathrm{min}^{-1} \mathrm{~kg}^{-1}$ ) and $2.65 \mathrm{ml} \mathrm{min}^{-1} \mathrm{~kg}^{-1}$ (95\% CI: 0.92 to $4.39 \mathrm{ml} \mathrm{min}^{-1} \mathrm{~kg}^{-1}$ ) at 6 and 12 months, respectively, with mild heterogeneity among studies (6-month follow-up, $I^{2}=55 \%, \quad P=0.003 ; 12$-month follow-up, $\left.I^{2}=22 \%, P=0.003\right)$ in four RCTs ${ }^{14-17}$ compared with placebo. There was a significant increase in $\mathrm{VO}_{2}$ at an AT of $2.01 \mathrm{ml} \mathrm{min}{ }^{-1} \mathrm{~kg}^{-1}$ (95\% CI: 1.03 to $2.99 \mathrm{ml} \mathrm{min}^{-1} \mathrm{~kg}^{-1}$ ) with mild heterogeneity overall $\left(P<0.0001, I^{2}=49.7 \%\right.$; Figure $\left.4 \mathrm{c}\right)$.

There was no significant decrease in peak VD/VT of 0.01 (95\% CI: -0.01 to 0.03$)$ at the acute duration $\left(P=0.49, I^{2}=89 \%\right)$ in the four RCTs $^{13,16,17,19}$ or one observational study ${ }^{27}$ compared with placebo. The pooling analysis showed that additional sildenafil therapy significantly decreased peak VD/VT at the 3-month follow-up (WMD: $-0.04, P<0.00001$; Figure 4d).

\section{Left ventricle ejection fraction}

There were no significant improvements in LVEF at the acute phase or 3 -month follow-up ( $I^{2}=67, P=0.74 ; I^{2}=50, P=0.34$, respectively) compared with placebo in four RCTs. ${ }^{13,14,16,17}$ However, sildenafil therapy was superior to placebo regarding LVEF at the 6-month follow-up with no heterogeneity (WMD: 4.35 , 95\% CI: 3.02 to 5.69 , $I^{2}=0, P<0.00001$; Figure 5).

\section{Quality of life}

QoL was assessed using a 16-question chronic HF questionnaire with answers scored from 1 (worse) to 7 (best). ${ }^{14,15,17}$ The QoL assessment documented a significant and sustained sildenafil-mediated improvement in breathlessness (WMD: 7.54 points, 95\% CI: 5.75 to 9.34, $P<0.00001$; WMD: 8.36 points, $95 \%$ CI: 5.86 to $10.86, P<0.00001$, respectively), fatigue (WMD: 3.20 points, $95 \% \mathrm{CI}$ : -0.01 to 6.41 , $P=0.05$; WMD: 5.80 points, 95\% CI: 2.92 to $8.68, P<0.0001$, respectively) and emotional function (WMD: 6.20 points, $95 \% \mathrm{CI}$ : 
a

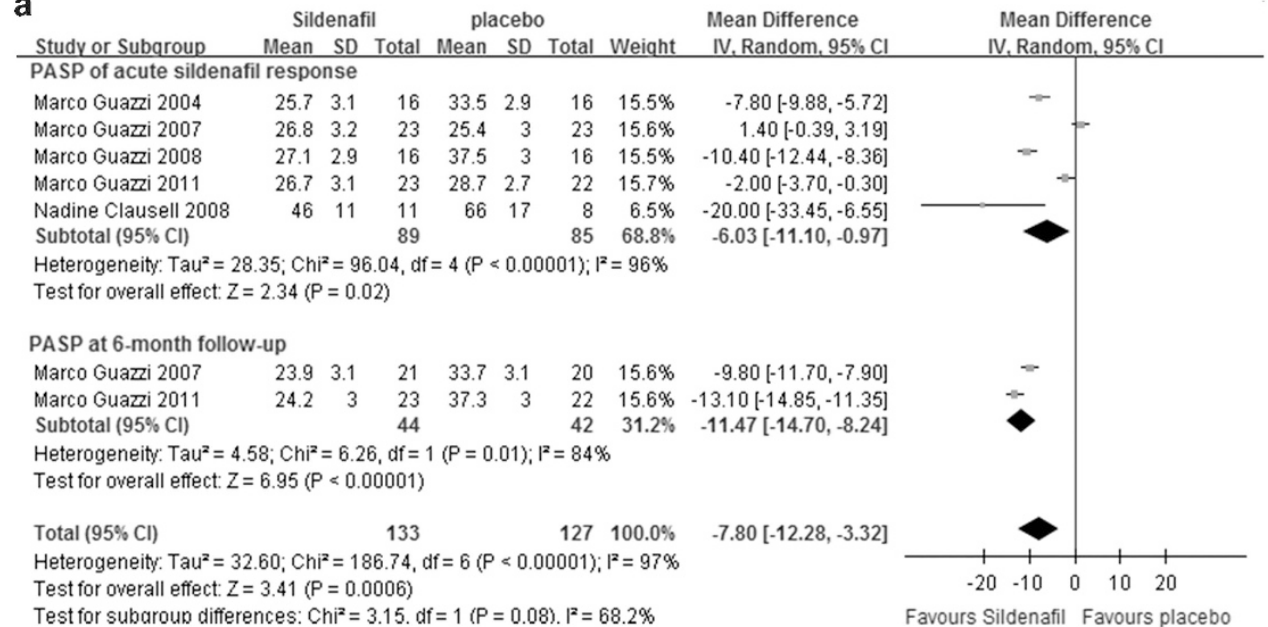

b

\begin{tabular}{|c|c|c|c|c|c|c|c|c|c|c|}
\hline \multirow{2}{*}{ Study or Subaroup } & \multirow{2}{*}{\multicolumn{2}{|c|}{$\begin{array}{l}\text { Sildenafil } \\
\text { Mean SD Total }\end{array}$}} & \multirow{2}{*}{\multicolumn{3}{|c|}{ placebo }} & & \multirow{3}{*}{$\begin{array}{c}\text { Mean Difference } \\
\text { IV. Fixed, } 95 \% \mathrm{Cl} \\
-300-41.1291\end{array}$} & \multirow{2}{*}{\multicolumn{3}{|c|}{$\begin{array}{l}\text { Mean Difference } \\
\text { IV, Fixed, } 95 \% \mathrm{Cl}\end{array}$}} \\
\hline & & & & & Total & Weight & & & & \\
\hline Marc J. Semigran 2007(1) & 28 & 17 & 31 & 3 & 17 & $93.7 \%$ & & -1 & & \\
\hline Marc J. Semigran 2008 & 28 & 15 & 31 & 11 & 15 & $6.3 \%$ & $-3.00[-9.60,3.60]$ & & & \\
\hline Total $(95 \% \mathrm{Cl})$ & & 32 & & & 32 & $100.0 \%$ & $-3.00[-4.66,-1.34]$ & & & \\
\hline \multicolumn{8}{|c|}{$\begin{array}{l}\text { Heterogeneity: } C \mathrm{Ch}^{2}=0.00, \mathrm{df}=1(P=1.00): \mathrm{l}^{2}=0 \% \\
\text { Test for overall effect } Z=3.54(P=0.0004)\end{array}$} & -5 & 0 & 5 \\
\hline
\end{tabular}

c

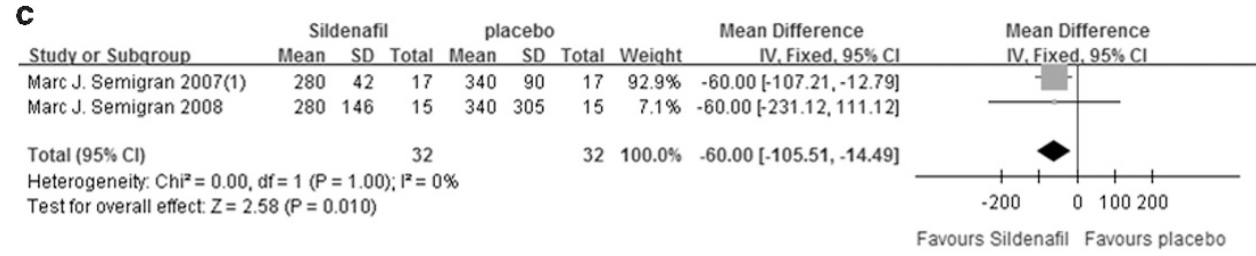

d

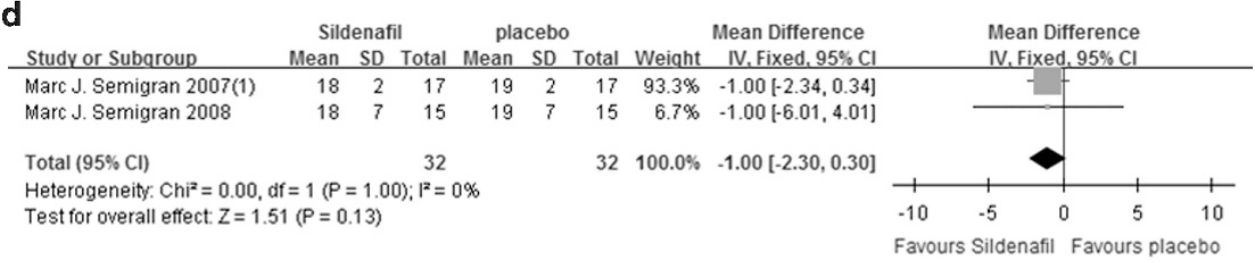

Figure 2 Forest plots for pulmonary hemodynamic measurements. (a) Pulmonary arterial systolic pressure (PASP) at 3-month follow-up. (b) Mean pulmonary artery pressure (mPAP) at 3-month follow-up. (c) Pulmonary vascular resistance (PVR) at 3-month follow-up. (d) Pulmonary capillary wedge pressure (PCWP) at 3-month follow-up. $\mathrm{Cl}$, confidence interval; IV, inverse variance.

3.71 to $8.70, P<0.00001$; WMD: 4.57 points, $95 \%$ CI: 1.53 to 7.62 , $P<0.003$, respectively) at the 6- and 12-month follow-ups, respectively. There was no significant heterogeneity among these studies (both breathlessness and emotional function, $I^{2}=0 \%$; fatigue, $I^{2}=50 \%$, overall; Figures $6 \mathrm{a}-\mathrm{c}$ ).

\section{DISCUSSION}

The present study demonstrated that PDE5 inhibitors (sildenafil) can be helpful in PH-LHD with HFrEF. Based on standard chronic HF therapy, additional sildenafil treatment significantly improves PASP regardless of whether as a short- or long-term treatment. Chronic therapy can improve mPAP and QoL in PH-LHD with HFrEF. Although there were no significant improvements in LVEF at the acute phase or at 3 months of follow-up, sildenafil therapy was superior to placebo regarding LVEF at the 6-month follow-up with no heterogeneity. There were significant improvements in peak $\mathrm{VO}_{2}, \mathrm{VE} / \mathrm{VCO}_{2}$ slope, $\mathrm{VO}_{2}$ at $\mathrm{AT}$ and peak VD/VT during chronic therapy only.
In our study, sildenafil significantly reduced the PASP, mPAP and PVR levels, although the PCWP level remained unchanged. We could not verify whether there were significant improvement in CO or cardiac index due to a lack of sufficient data to evaluate the effects. $^{15,27}$ Sildenafil-lowering PAP and PVR in our study was consistent with the findings of Huang et al. ${ }^{26}$ However, our research showed that sildenafil not only reduced PASP within $1-2 \mathrm{~h}$ after administration but also generates more obvious effect at 6 months of follow-up in patients with HFrEF, different from Huang et al., ${ }^{26}$ who studied the chronic effect of sildenafil on mPAP and PVR by mixing different follow-up lengths. We believe that the chronic effects of sildenafil should be analyzed stepwise by subgroup meta-analysis according to follow-up time because the long-term effects of the drug might be different at different times. We evaluated only changes in mPAP and PVR at 3 months of follow-up due to a lack of sufficient data to evaluate the effects at 6 or 12 months. 


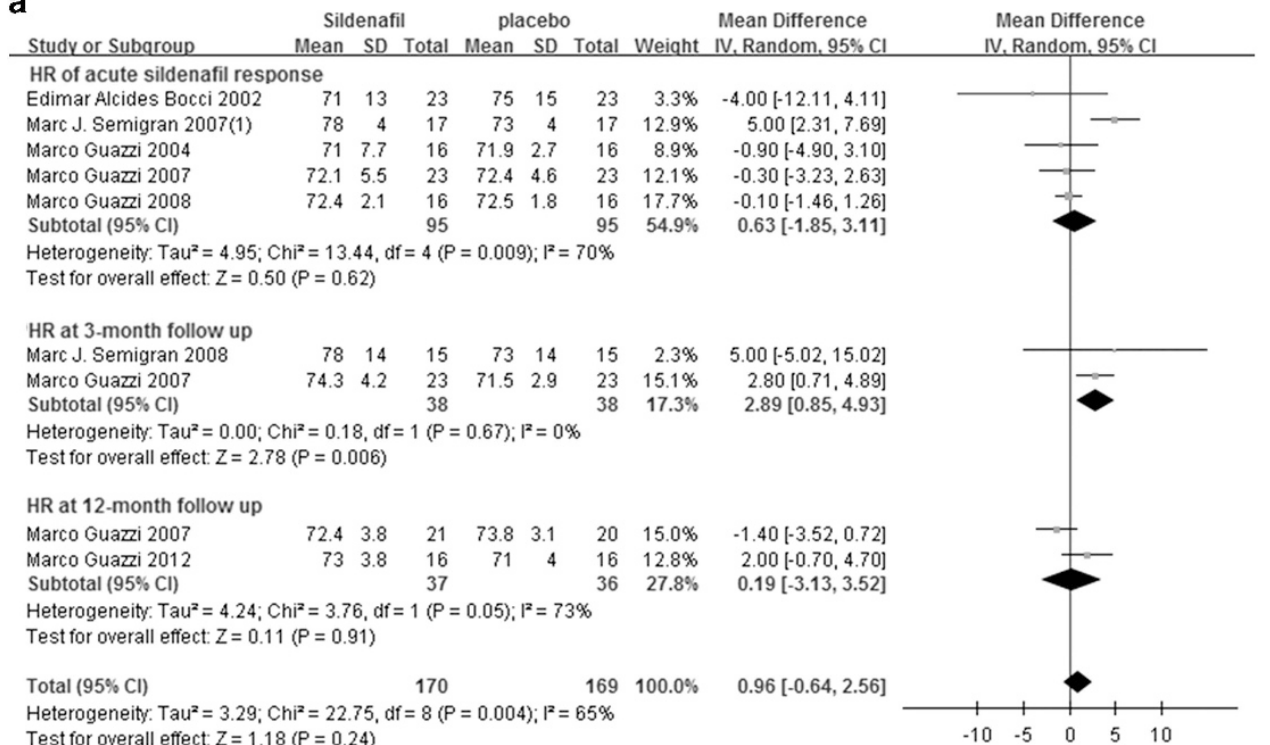

Heterogeneity. Tau $^{2}=3.29 ; \mathrm{Chi}^{2}=22.75, \mathrm{df}=8(\mathrm{P}=0.004) ; \mathrm{I}^{2}=65 \%$
Test for overall effect $\mathrm{Z}=1.18(\mathrm{P}=0.24)$

Test for subaroun differences: $\mathrm{Chi}^{2}=2.81 . \mathrm{df}=2(\mathrm{P}=0.25) . \mathrm{I}^{2}=28.9 \%$

Favours Sildenafil Favours placebo

b

Sildenafil Placebo Mean Difference

Study or Subqroup Mean SD Total Mean SD Total Weight IV. Random. $95 \% \mathrm{Cl}$ SBP of acute sildenafil response

$108 \quad 17$

Marco Guazzi 2004

Marco Guazzi 2007

$\begin{array}{rr}108 \quad 17 \\ 115.6 & 9\end{array}$

Marco Guazzi 2008

$115.6 \quad 9$

$\begin{array}{lll}23 & 116 & 15\end{array}$

$23 \quad 5.0 \%$

Marco Guazzi 2011

121.37

$\begin{array}{lll}122.3 & 3.3\end{array}$

$\begin{array}{lll}16 & 121 & 6.8\end{array}$

$\begin{array}{ll}16 & 11.0 \% \\ 23 & 15.1 \%\end{array}$

$\begin{array}{lllllll}122.3 & 3.3 & 16 & 123.8 & 3.1 & 16 & 24.6 \% \\ 108.4 & 7.7 & 23 & 105.2 & 9.5 & 22 & 12.3 \%\end{array}$

Subtotal $(95 \% \mathrm{Cl})$

$\begin{array}{ll}16 & 24.6 \% \\ 22 & 12.3 \%\end{array}$

101

$=0.11): 1^{2}=47 \%$

Heterogeneity. $\mathrm{Tau}^{2}=4.44 ; \mathrm{Chi}^{2}=7.49, \mathrm{df}$

SBP at 6-month follow-up

Marco Guazzi 2011

$124.4 \quad 4.5$

$4.5 \quad 21$

.00 $-17.27,1.27$

$-0.10[-4.32,4.12$

$-1.50[-3.72,0.72]$

$3.20[-1.87,8.27]$

Mean Difference

Subtotal $(95 \% \mathrm{Cl})$

111.38 .9

Heterogeneity $\mathrm{Tau}^{2}=0.00 ; \mathrm{Chi}^{2}=0.09, \mathrm{dr}$

Test for overall effect: $Z=1.41(P=0.16)$

Total $(95 \% \mathrm{Cl})$

145

$\begin{array}{lllll}1 & 122.2 & 5.8 & 20 & 19.6 \%\end{array}$

$23 \quad 12.4 \%$ 4

$43 \quad 32.0 \%$

$1.30[-3.73,6.33]$

$1.94[-0.75,4.63]$

Heterogeneity: $\mathrm{Tau}^{2}=4.08 ; \mathrm{Chi}^{2}=11.63, \mathrm{df}=6(\mathrm{P}=0.07) ; \mathrm{I}^{2}=48 \%$

Test for overall effect: $Z=0.34(P=0.73)$

Test for subaroun differences: $\mathrm{Chi}^{2}=2.96 . \mathrm{df}=1(\mathrm{P}=0.09) . \mathrm{I}^{2}=66.2 \%$

$100.0 \% \quad-0.39[-2.64,1.86]$

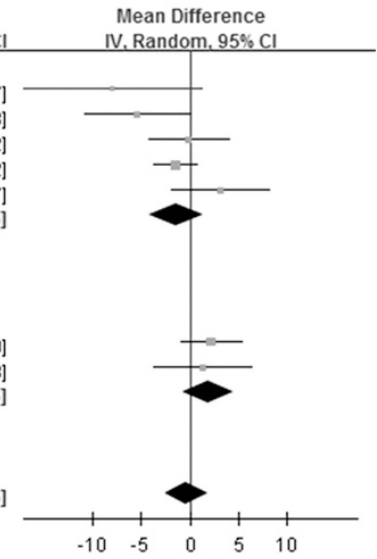

C

Sildenafil placebo Mean Difference

Favours Sildenafil Favours placebo

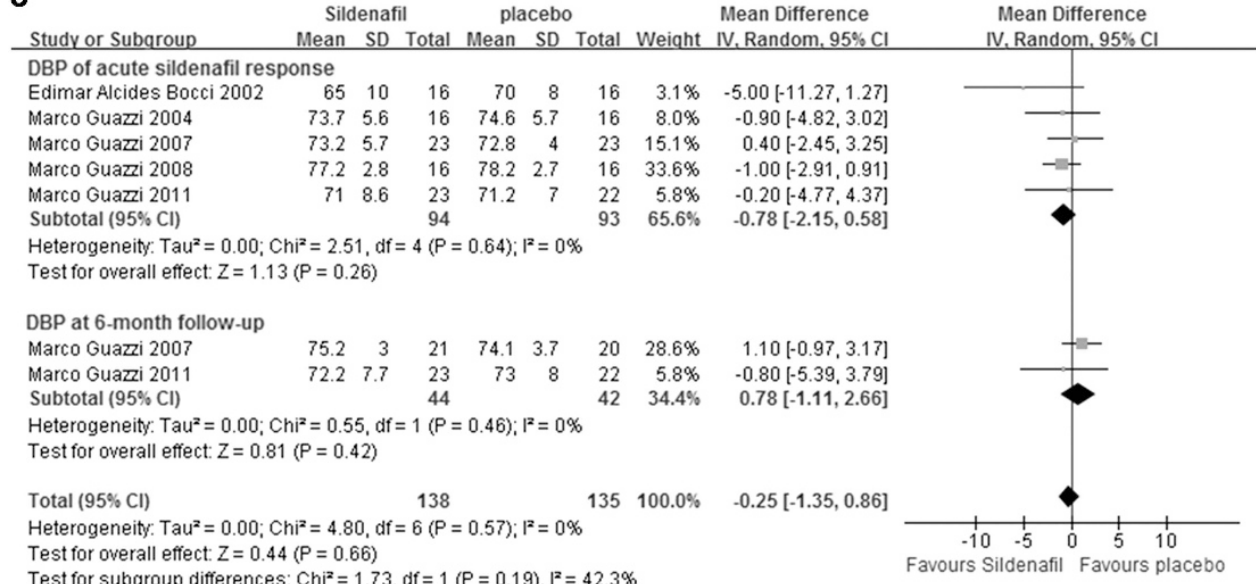

Figure 3 Forest plots for pulmonary systematic hemodynamic measurements. (a) Heart rate (HR). (b) Systolic blood pressure (SBP). (c) Diatolic blood pressure (DBP). $\mathrm{Cl}$, confidence interval; IV, inverse variance. 
Systemic BP in $\mathrm{PH}$ patients tends to be low. In this meta-analysis, sildenafil produced a selective response in the pulmonary vessels without significant changes in the left heart hemodynamic variables, not only in the acute phase but also in the chronic phase. The study by
Bocchi et al. ${ }^{19}$ showed that sildenafil treatment did not result in a greater reduction in systemic BP or in patients receiving nitrates for pulmonary congestion. Sildenafil therapy also had no effect on HR.
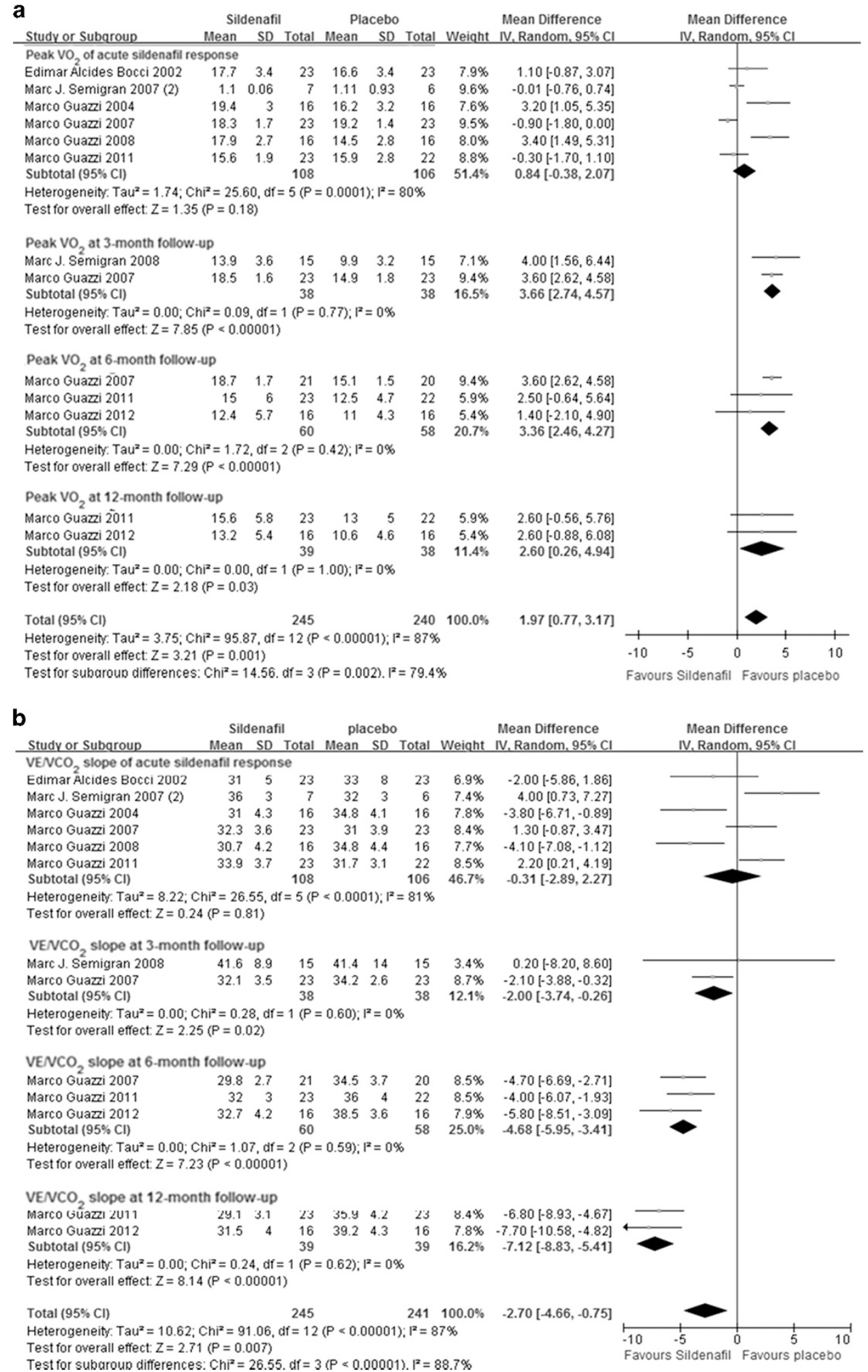

Figure 4 Forest plots for cardiopulmonary exercise testing. (a) Oxygen consumption at peak exercise (peak $\mathrm{VO}_{2}$ ). (b) The efficiency of ventilatory response to carbon dioxide output ( $\mathrm{VEN} / \mathrm{NO}_{2}$ slope). (c) $\mathrm{VO}_{2}$ at anaerobic threshold (AT) $\left(\mathrm{VO}_{2}\right.$ at $\mathrm{AT}$ ). (d) The ratio of dead space to tidal volume at peak exercise (peak $\mathrm{VD} / \mathrm{VT}$ ). $\mathrm{Cl}$, confidence interval; IV, inverse variance. 


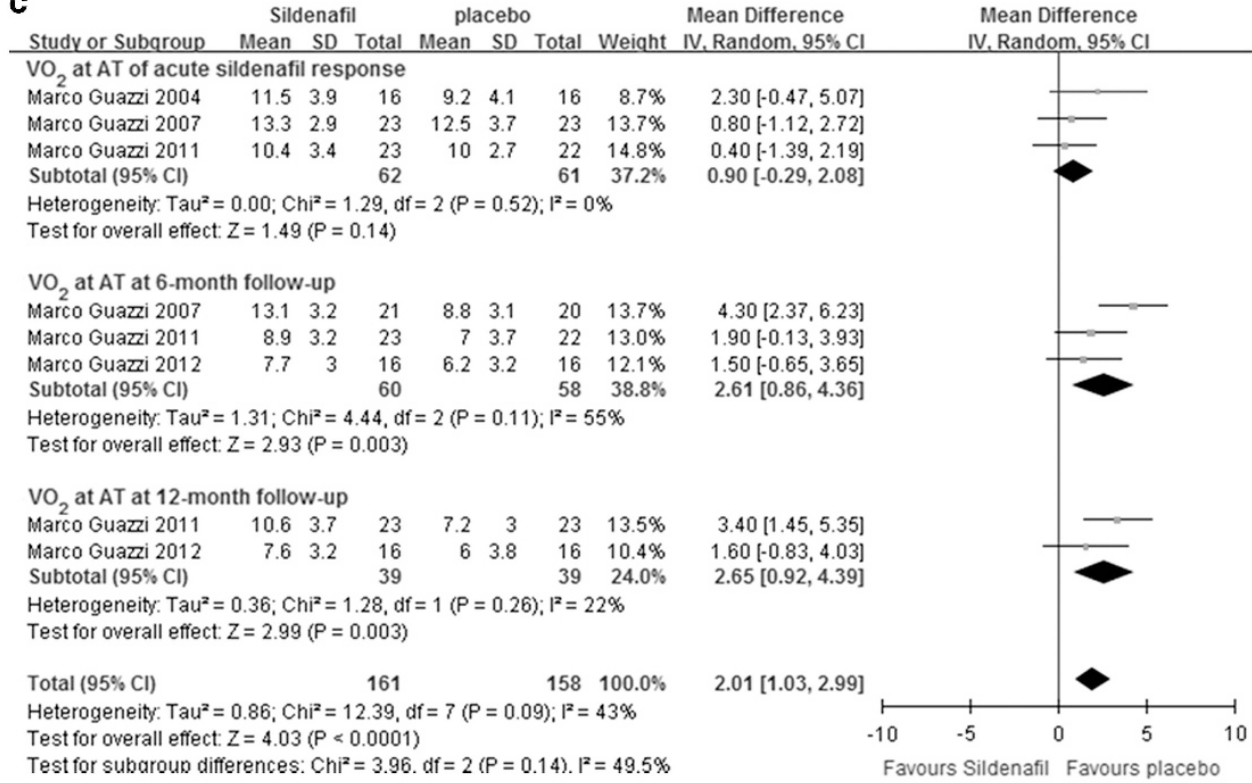

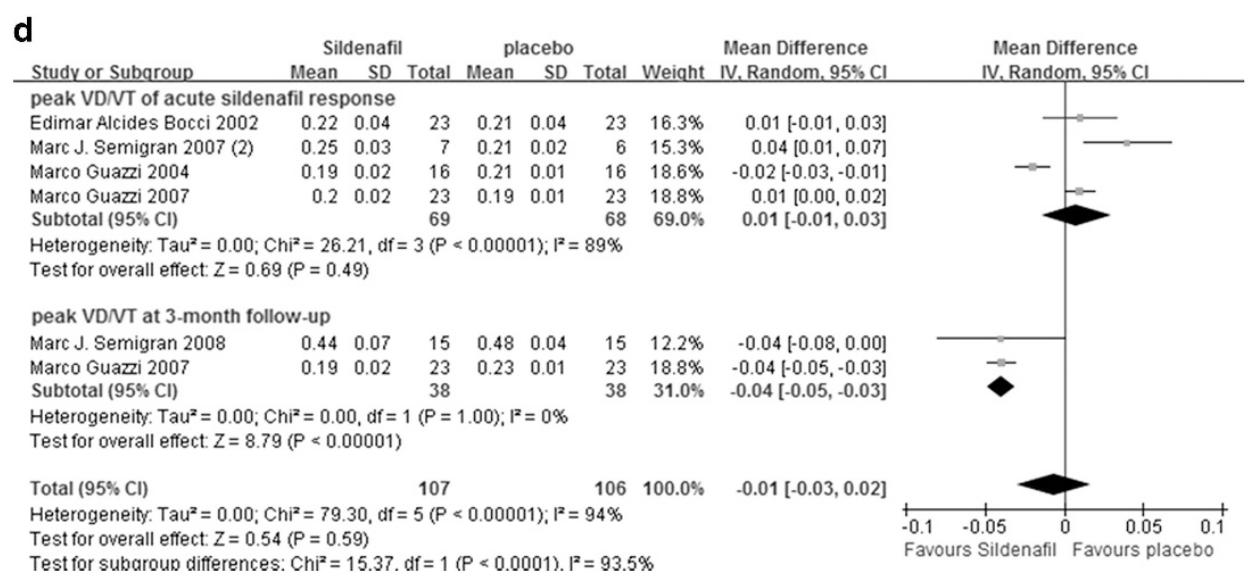

Figure 4 continued

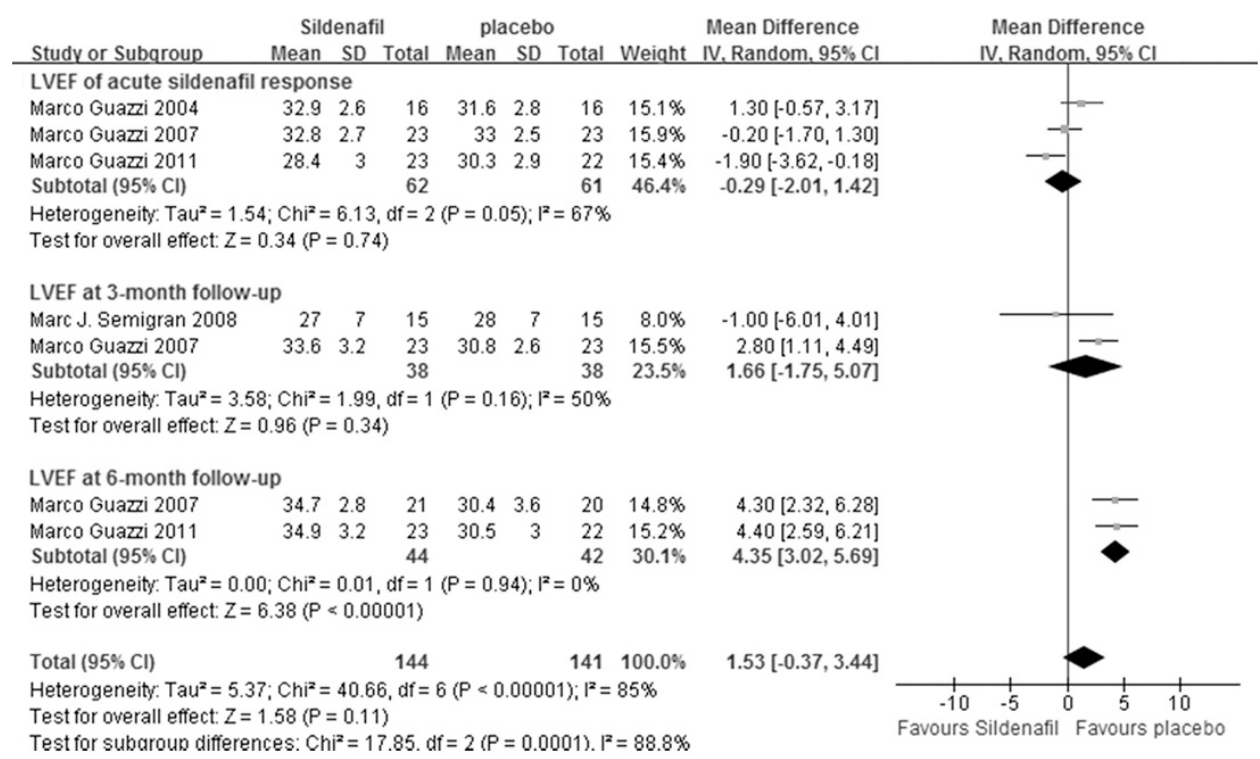

Figure 5 Forest plots for left ventricle ejection fraction. $\mathrm{Cl}$, confidence interal; IV, inverse variance. 
a

\begin{tabular}{|c|c|c|c|c|c|c|c|c|c|c|c|c|}
\hline & Sild & lenaf & & & acebc & & & Mean Difference & & Mean & Difference & \\
\hline Breathlessness at & -month & follo & N-up & & & & & & & & & \\
\hline Marco Guazzi 2007 & 24.1 & 5.2 & 21 & 17.2 & 45 & 20 & $241 \%$ & 600130300071 & & & & \\
\hline Marco Guazzi 2011 & 31.4 & 5.2 & 23 & 23.1 & 5 & 22 & $24.0 \%$ & $8.30[5.32,11.28]$ & & & & \\
\hline Marco Guazzi 2012 & 23 & 4 & 16 & 15.6 & 5.8 & 16 & $17.9 \%$ & $7.40[3.95,10.85]$ & & & & \\
\hline Subtotal $(95 \% \mathrm{Cl})$ & & & 60 & & & 58 & $65.9 \%$ & $7.54[5.75,9.34]$ & & & & \\
\hline $\begin{array}{l}\text { Heterogeneity } \mathrm{Tau}^{2} \\
\text { Test for overall effec }\end{array}$ & $\begin{array}{l}0.00 ; \mathrm{Cr} \\
\mathrm{Z}=8.23\end{array}$ & $\begin{array}{l}h^{2}= \\
(P<\end{array}$ & $\begin{array}{l}43, \mathrm{df} \\
0.0000\end{array}$ & $\begin{array}{l}=2(P \\
1)\end{array}$ & $=0.8$ & )$: 1^{2}=0$ & & & & & & \\
\hline Breathlessness at & 2-montr & h foll & ow-up & & & & & & & & & \\
\hline Marco Guazzi 2011 & 31.5 & 4.9 & 23 & 22.9 & 6 & 22 & $20.7 \%$ & $8.60[5.39,11.81]$ & & & & \\
\hline Marco Guazzi 2012 & 24.2 & 6 & 16 & 16.2 & 5.5 & 16 & $13.4 \%$ & $8.00[4.01,11.99]$ & & & & \\
\hline Subtotal $(95 \% \mathrm{Cl})$ & & & 39 & & & 38 & $34.1 \%$ & $8.36[5.86,10.86]$ & & & & \\
\hline $\begin{array}{l}\text { Heterogeneity. Tau } \\
\text { Test for overall effec }\end{array}$ & $\begin{array}{l}0.00 ; \mathrm{Cr} \\
\mathrm{Z}=6.56\end{array}$ & $\begin{array}{l}\mathrm{h}^{2}= \\
(\mathrm{P}<\end{array}$ & $\begin{array}{l}.05, \mathrm{df} \\
0.0000\end{array}$ & $\begin{array}{l}=1(P \\
1)\end{array}$ & $=0.8$ & $; 1^{2}=c$ & & & & & & \\
\hline Total $(95 \% \mathrm{Cl})$ & & & 99 & & & 96 & $100.0 \%$ & $7.82[6.36,9.28]$ & & & & \\
\hline $\begin{array}{l}\text { Heterogeneity. Tau } \\
\text { Test for overall effec }\end{array}$ & $\begin{array}{l}0.00 ; C r \\
z=10.5\end{array}$ & $\begin{array}{l}\mathrm{h}^{2}= \\
1(\mathrm{P} .\end{array}$ & $\begin{array}{l}76, \mathrm{df} \\
0.000\end{array}$ & $\begin{array}{l}=4(P \\
01)\end{array}$ & & & & & -10 & -5 & 0 & 10 \\
\hline
\end{tabular}

b

$\begin{array}{ccc}\text { Sildenafil } & \text { Placebo } & \text { Mean Difference } \\ \text { Study or Subqroup } & \text { Mean SD Total Mean SD Total Weight IV. Random. } 95 \% \mathrm{Cl}\end{array}$ Fatigue at 6-month follow-up

$\begin{array}{lllllllll}\text { Marco Guazzi } 2007 & 21.5 & 5.4 & 21 & 20.7 & 6.2 & 20 & 20.6 \% & 0.80[-2.77,4.37]\end{array}$

$\begin{array}{lrrrrrrrr}\text { Marco Guazzi } 2011 & 28.4 & 5 & 23 & 22.4 & 6.1 & 22 & 22.4 \% & 6.00[2.73,9.27]\end{array}$

$\begin{array}{lllllllll}\text { Marco Guazzi } 2012 & 21 & 6 & 16 & 18.5 & 6 & 16 & 17.6 \% & 2.50[-1.66,6.66]\end{array}$

$\begin{array}{lllll}\text { Subtotal }(95 \% \mathrm{Cl}) & 60 & 58 & 60.6 \% & 3.20[-0.01,6.41]\end{array}$

Heterogeneity. $\mathrm{Tau}^{2}=4.58 ; \mathrm{Chi}^{2}=4.65, \mathrm{df}=2(\mathrm{P}=0.10) ; \mathrm{I}^{2}=57 \%$

Test for overall effect: $Z=1.95(P=0.05)$

Fatigue at 12-month follow-up

$\begin{array}{lrrrrrrrr}\text { Marco Guazzi } 2011 & 29 & 5 & 23 & 22 & 6.2 & 22 & 22.2 \% & 7.00[3.70,10.30] \\ \text { Marco Guazi } 2012 & 23 & 6.2 & 16 & 19 & 6 & 16 & 17.2 \% & 4.00[-0.23,8.23]\end{array}$

$\begin{array}{lllllllll}\text { Marco Guazzi } 2012 & 23 & 6.2 & 16 & 19 & 6 & 16 & 17.2 \% & 4.00[-0.23,8.23] \\ \text { Subtotal }(95 \% \mathrm{Cl}) & & & 39 & & & 38 & 39.4 \% & 5.80[2.92,8.68]\end{array}$

Heterogeneity $\mathrm{Tau}^{2}=0.76 ; \mathrm{Chi}^{2}=1.20, \mathrm{df}=1(\mathrm{P}=0.27) ; \mathrm{I}^{2}=17 \%$

Test for overall effect: $Z=3.95(P<0.0001)$

Total $(95 \% \mathrm{Cl}) \quad 99 \quad 96 \quad 100.0 \%$

Heterogeneity: Tau $^{2}=3.53 ; \mathrm{Chi}^{2}=8.05, \mathrm{df}=4(\mathrm{P}=0.09) ; \mathrm{I}^{2}=50 \%$

Test for overall effect: $Z=3.53(P=0.0004)$

Test for subaroun differences: $\mathrm{Chi}^{2}=1.40 . \mathrm{df}=1(\mathrm{P}=0.24) . \mathrm{I}^{2}=28.7 \%$

$5.80[2.92,8.68]$

$4.19[1.86,6.52]$

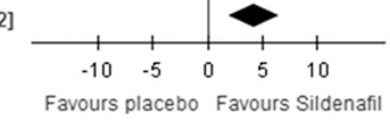

C

\begin{tabular}{|c|c|c|c|c|c|c|c|c|c|c|c|c|}
\hline \multirow{2}{*}{ Study or Subqroup } & \multicolumn{3}{|c|}{ Sildenafil } & \multicolumn{3}{|c|}{ Placebo } & Weight & $\begin{array}{l}\text { Mean Difference } \\
\text { IV. Random, } 95 \% \mathrm{Cl}\end{array}$ & \multicolumn{3}{|c|}{$\begin{array}{l}\text { Mean Difference } \\
\text { IV, Random, } 95 \% \mathrm{Cl}\end{array}$} & \\
\hline & \multirow{2}{*}{\multicolumn{11}{|c|}{ Emotional function at 6-month follow-up }} & \\
\hline Marco Guazzi 2007 & 34.1 & & 21 & 25 & 8.4 & 20 & $15.1 \%$ & $9.10[4.13,14.07]$ & & & & \\
\hline Marco Guazzi 2011 & 36.8 & 6.6 & 23 & 31.7 & 6.3 & 22 & $26.2 \%$ & $5.10[1.33,8.87]$ & & & & \\
\hline Marco Guazzi 2012 & 31.4 & 6 & 16 & 26 & 6.9 & 16 & $18.6 \%$ & $5.40[0.92,9.88]$ & & & & \\
\hline Subtotal $(95 \% \mathrm{Cl})$ & & & 60 & & & 58 & $59.9 \%$ & $6.20[3.71,8.70]$ & & & & \\
\hline \multicolumn{13}{|c|}{$\begin{array}{l}\text { Heterogeneity: } \mathrm{Tau}^{2}=0.00 ; \mathrm{Chi}^{2}=1.76, \mathrm{df}=2(P=0.42) ; \mathrm{I}^{2}=0 \% \\
\text { Test for overall effect: } Z=4.87(P<0.00001)\end{array}$} \\
\hline \multicolumn{13}{|c|}{ Emotional function at 12-month follow-up } \\
\hline Marco Guazzi 2011 & 36.1 & 6.7 & 23 & 32.2 & 7 & 22 & $23.2 \%$ & $3.90[-0.11,7.91]$ & & & & \\
\hline Marco Guazzi 2012 & 31 & 7.3 & 16 & 25.5 & 6.2 & 16 & $16.9 \%$ & $5.50[0.81,10.19]$ & & & & \\
\hline Subtotal $(95 \% \mathrm{Cl})$ & & & 39 & & & 38 & $40.1 \%$ & $4.57[1.53,7.62]$ & & & & \\
\hline \multicolumn{13}{|c|}{$\begin{array}{l}\text { Heterogeneity. Tau }{ }^{2}=0.00 ; \mathrm{Chi}^{2}=0.26, \mathrm{df}=1(P=0.61) ; \mathrm{I}^{2}=0 \% \\
\text { Test for overall effect: } Z=2.94(P=0.003)\end{array}$} \\
\hline Total $(95 \% \mathrm{Cl})$ & & & 99 & & & 96 & $100.0 \%$ & $5.55[3.62,7.48]$ & & & & \\
\hline \multicolumn{9}{|c|}{$\begin{array}{l}\text { Heterogeneity. } \mathrm{Tau}^{2}=0.00 ; \mathrm{Chi}^{2}=2.67, \mathrm{df}=4(P=0.61) ; \mathrm{I}^{2}=0 \% \\
\text { Test for overall effect } Z=5.63(P<0.00001)\end{array}$} & -10 & -5 & 0 & 10 \\
\hline
\end{tabular}

Figure 6 Forest plots for quality of life. (a) Breathlessness. (b) Fatigue. (c) Emotional function. Cl, confidence interval; IV, inverse variance.

Our meta-analysis showed that one-time or chronic administration of sildenafil to patients with $\mathrm{HFrEF}$ improved peak $\mathrm{VO}_{2}, \mathrm{VO}_{2}$ at $\mathrm{AT}$ and reduced $\mathrm{VE} / \mathrm{VCO}_{2}$ slope (Figures $4 \mathrm{a}-\mathrm{c}$ ). The changes in peak $\mathrm{VO}_{2}, \mathrm{VO}_{2}$ at $\mathrm{AT}$ or $\mathrm{VE} / \mathrm{VCO}_{2}$ slope at 3, 6 and 12 months were all consistent. Exercise capacity, as measured by peak $\mathrm{VO}_{2}$, is a well-established independent predictor of prognosis in patients with advanced systolic $\mathrm{HF}^{31-33}$ The improvement in peak $\mathrm{VO}_{2}$ observed with sildenafil treatment in this study was attributable to augmentation of CO. Several mechanisms could account for this improvement in CO. Sildenafil improved RV systolic function, as reflected by an increase in RVEF, measured with radionuclide ventriculography. ${ }^{12}$

The correlation between the changes in peak $\mathrm{VO}_{2}$ with sildenafil treatment and baseline PAP also suggests that a reduction in RV afterload might have mediated the improvement in exercise capacity. This augmentation in RV function was likely attributable to afterload reduction because sildenafil reduces PVR. ${ }^{27}$

An exaggerated ventilatory response to exertion is perceived as a sense of breathlessness and is objectified by an increase in $\mathrm{VE} / \mathrm{VCO}_{2}$ slope. Hyperventilation can help in maintaining the normal alveolar 
tension of oxygen, which, however, accelerates exhaustion of the ventilatory reserve and an interruption of exercise. Several mechanisms could explain the basis of hyperventilation, that is, hypoperfusion of the lungs, enlarged dead space of the lungs, bicarbonate buffering of accumulated lactic acid, pulmonary interstitial space distension and abnormal drive from the reflexogenic areas of the cardiovascular system, ${ }^{34}$ skeletal muscle over-signaling and increased passage of fluid from the lungs intravascularly to the interstitial phase because of an excessive increase of the pulmonary capillary pressure on exertion. ${ }^{18} \mathrm{It}$ is inferable that an improvement in exercise pulmonary hemodynamics, $\mathrm{CO}$ and peripheral perfusion could attenuate many of these disorders, augmenting exercise capacity and reducing hyperventilation.

Chronically elevated resting left-sided filling pressures might contribute to inadequate pulmonary vasodilation and high VD/VT. ${ }^{13}$ Inefficient ventilation in patients with advanced HF has been ascribed to abnormalities in gas exchange (higher VD/VT), as well as to an independent, 'primary' drive to hyperventilation that results in low $\mathrm{PaCO}_{2}{ }^{35}$ Lewis et al. ${ }^{13}$ observed both a positive correlation between PVR and VD/VT at peak exercise and a negative correlation between PVR and arterial $\mathrm{PaCO}_{2}$ at peak exercise. Peak VD/VT had a positive correlation with PVR. In our study, although peak VD/VT remained unchanged at the acute phase, peak VD/VT was significantly reduced at 3 months of follow-up.

Some studies have reported that sildenafil intake was associated with increases in peak RER, ${ }^{16,27}$ peak workload ${ }^{16}$ and $\Delta \mathrm{VO}_{2} / \Delta \mathrm{WR} ;{ }^{16,18}$ however, we noted that sildenafil did not produce significant changes in peak RER, peak workload or $\Delta \mathrm{VO}_{2} / \Delta \mathrm{WR}$ (Supplementary Figures $3 \mathrm{~A}-\mathrm{C}$ ).

Some studies in animals and humans with HF have indicated that PDE5 inhibition does not alter myocardial contractility. ${ }^{36-38}$ Nagendran et al. ${ }^{38}$ suggested that PDE5 inhibition could increase RV contractility in the setting of RV hypertrophy. In the study by Huang et al., ${ }^{26}$ additional sildenafil intervention enhanced LV function in HFrEF patients with long-term treatment of sildenafil. However, in our meta-analysis, we revealed that sildenafil improved LVEF at 6 months of chronic therapy. One-time or 3-month administration of sildenafil did not improve LVEF (Figure 5). We conclude that LVEF, as measured by echocardiography, might require a longer time to show significant change.

Sildenafil has been shown to be effective as a pulmonary vasodilator through selective inhibition of PDE5 in patients with pulmonary pressure increases secondary to chronic HF. These effects are explainable on the basis of the mechanism of action of the drug. Sildenafil, by slowing the degradation of cGMP, potentiates the effects of limited nitric oxide production in the presence of endothelial dysfunction, particularly in HF. ${ }^{39}$ Reductions in PAP and PVR lead to improved LVEF, increased CO, enhanced exercise performance ${ }^{27}$ and a shortened diffusion path between the alveoli and capillaries because of diminished fluid flux transition into the alveolar interstitial space. ${ }^{16}$

The QoL assessment documented a significant and sustained sildenafil-mediated improvement in breathlessness, fatigue and emotional function.

\section{Limitations}

First, the number of studies was limited. Second, all of the included studies had small sample sizes, were single-center studies; multicenter clinical trials with larger sample size in PH-LHD with HFrEF are currently under way, including Sildenafil in Heart Failure, ${ }^{40}$ and they might address these issues. Third, the included population had different characteristics, such as the patient's age, sex, treatment history and so on. The population with HF is more heterogeneous than in PAH, resulting in complex polypharmacy. Patients should be defined in future large-scale clinical trials. Fourth, the longest followup duration was only 12 months. We expect longer observation times in future studies. Finally, although encouraging, the results should be treated with caution because the studies were single-center studies and used a range of doses of sildenafil (from 25 to $75 \mathrm{mg}$ tid) that were consistently higher than the doses approved to treat PAH.

\section{CONCLUSIONS}

The present meta-analysis suggested that sildenafil improved pulmonary hemodynamics, both at the acute phase and over long-term follow-up, without changes in HR or systematic blood pressure. Sildenafil therapy also improves CPET measurements, LVEF and QoL, during both the acute phase and over long-term follow-up. Although it might be challenging, carefully controlled trials with larger and subset populations, ideally in similar proof concept and phase III studies (that is, identified by invasive hemodynamic status, performed in the same clinical setting with patients on optimized and stable therapy), are needed. These studies will show the exact effects of sildenafil in such a patient group.

\section{CONFLICT OF INTEREST}

The authors declare no conflict of interest.

\section{ACKNOWLEDGEMENTS}

This research was supported by the Program of the Shanghai Municipal Commission of Health and Family Planning (20144Y0196), the Young Foundation Sanitary Bureau of Shanghai (grant no. 20124Y117) and College Project for Tongji University (1511219015). The sponsors had no involvement in the study design, data analysis, data interpretation or writing and revision of the report.

Author contributions: All the authors take responsibility for all aspects of the reliability and freedom from bias of the data presented and their discussed interpretation.

1 Fang JC, DeMarco T, Givertz MM, Borlaug BA, Lewis GD, Rame JE, Gomberg-Maitland M, Murali S, Frantz RP, McGlothlin D, Horn EM, Benza RL. World Health Organization Pulmonary Hypertension group 2: pulmonary hypertension due to left heart disease in the adult-a summary statement from the Pulmonary Hypertension Council of the International Society for Heart and Lung Transplantation. J Heart Lung Transplant 2012; 31: 913-933.

2 Guazzi M, Borlaug BA. Pulmonary hypertension due to left heart disease. Circulation 2012; 126: 975-990.

3 Galie N, Hoeper MM, Humbert M, Torbicki A, Vachiery JL, Barbera JA, Beghetti M, Corris P, Gaine S, Gibbs JS, Gomez-Sanchez MA, Jondeau G, Klepetko W, Opitz C Peacock A, Rubin L, Zellweger M, Simonneau G. Guidelines for the diagnosis and treatment of pulmonary hypertension: the Task Force for the Diagnosis and Treatment of Pulmonary Hypertension of the European Society of Cardiology (ESC) and the European Respiratory Society (ERS), endorsed by the International Society of Heart and Lung Transplantation (ISHLT). Eur Heart J 2009; 30: 2493-2537.

4 Vachiery JL, Adir Y, Barbera JA, Champion H, Coghlan JG, Cottin V, De Marco T, Galie N, Ghio S, Gibbs JS, Martinez F, Semigran M, Simonneau G, Wells A, Seeger W. Pulmonary hypertension due to left heart diseases. J Am Coll Cardiol 2013; 62 D100-D108.

5 Naeije R, Vachiery JL, Yerly P, Vanderpool R. The transpulmonary pressure gradient for the diagnosis of pulmonary vascular disease. Eur Respir J 2013; 41: 217-223.

6 Wei L, Zhu W, Xia L, Yang Y, Liu H, Shen J, Zhu J, Xu Y, Yang Z, Wang C. Therapeutic effect of eNOS-transfected endothelial progenitor cells on hemodynamic pulmonary arterial hypertension. Hypertens Res 2013; 36: 414-421.

7 Tedford RJ, Hassoun PM, Mathai SC, Girgis RE, Russell SD, Thiemann DR Cingolani $\mathrm{OH}$, Mudd JO, Borlaug BA, Redfield MM, Lederer DJ, Kass DA. Pulmonary capillary wedge pressure augments right ventricular pulsatile loading. Circulation 2012 ; 125: 289-297.

8 Ghio S, Gavazzi A, Campana C, Inserra C, Klersy C, Sebastiani R, Arbustini E, Recusani F, Tavazzi L. Independent and additive prognostic value of right ventricular systolic function and pulmonary artery pressure in patients with chronic heart failure. J Am Coll Cardiol 2001; 37: 183-188. 
9 Schwartzenberg S, Redfield MM, From AM, Sorajja P, Nishimura RA, Borlaug BA. Effects of vasodilation in heart failure with preserved or reduced ejection fraction implications of distinct pathophysiologies on response to therapy. J Am Coll Cardiol 2012; 59: 442-451.

10 Lam CS, Roger VL, Rodeheffer RJ, Borlaug BA, Enders FT, Redfield MM. Pulmonary hypertension in heart failure with preserved ejection fraction: a community-based study. J Am Coll Cardiol 2009; 53: 1119-1126.

11 Guazzi M, Vicenzi M, Arena R, Guazzi MD. Pulmonary hypertension in heart failure with preserved ejection fraction: a target of phosphodiesterase- 5 inhibition in a 1-year study. Circulation 2011; 124: 164-174.

12 Lewis GD, Shah R, Shahzad K, Camuso JM, Pappagianopoulos PP, Hung J, Tawakol A, Gerszten RE, Systrom DM, Bloch KD, Semigran MJ. Sildenafil improves exercise capacity and quality of life in patients with systolic heart failure and secondary pulmonary hypertension. Circulation 2007; 116: 1555-1562.

13 Lewis GD, Shah RV, Pappagianopolas PP, Systrom DM, Semigran MJ. Determinants of ventilatory efficiency in heart failure: the role of right ventricular performance and pulmonary vascular tone. Circ Heart Fail 2008; 1: 227-233.

14 Guazzi M, Vicenzi M, Arena R, Guazzi MD. PDE5 inhibition with sildenafil improves left ventricular diastolic function, cardiac geometry, and clinical status in patients with stable systolic heart failure: results of a 1-year, prospective, randomized, placebocontrolled study. Circ Heart Fail 2011; 4: 8-17.

15 Guazzi M, Vicenzi M, Arena R. Phosphodiesterase 5 inhibition with sildenafil reverses exercise oscillatory breathing in chronic heart failure: a long-term cardiopulmonary exercise testing placebo-controlled study. Eur J Heart Fail 2012; 14: 82-90.

16 Guazzi M, Tumminello G, Di Marco F, Fiorentini C, Guazzi MD. The effects of phosphodiesterase-5 inhibition with sildenafil on pulmonary hemodynamics and diffusion capacity, exercise ventilatory efficiency, and oxygen uptake kinetics in chronic heart failure. J Am Coll Cardiol 2004; 44: 2339-2348.

17 Guazzi M, Samaja M, Arena R, Vicenzi M, Guazzi MD. Long-term use of sildenafil in the therapeutic management of heart failure. J Am Coll Cardiol 2007; 50: 2136-2144.

18 Guazzi M, Casali M, Berti F, Rossoni G, Colonna VD, Guazzi MD. Endothelium-mediated modulation of ergoreflex and improvement in exercise ventilation by acute sildenafil in heart failure patients. Clin Pharmacol Ther 2008; 83: 336-341.

19 Bocchi EA, Guimaraes G, Mocelin A, Bacal F, Bellotti G, Ramires JF. Sildenafil effects on exercise, neurohormonal activation, and erectile dysfunction in congestive heart failure: a double-blind, placebo-controlled, randomized study followed by a prospective treatment for erectile dysfunction. Circulation 2002; 106: 1097-1103.

20 Behling A, Rohde LE, Colombo FC, Goldraich LA, Stein R, Clausell N. Effects of 5'-phosphodiesterase four-week long inhibition with sildenafil in patients with chronic heart failure: a double-blind, placebo-controlled clinical trial. J Card Fail 2008; 14: 189-197.

21 Thenappan T, Shah SJ, Gomberg-Maitland M, Collander B, Vallakati A, Shroff P, Rich S. Clinical characteristics of pulmonary hypertension in patients with heart failure and preserved ejection fraction. Circ Heart Fail 2011; 4: 257-265.

22 Robbins IM, Newman JH, Johnson RF, Hemnes AR, Fremont RD, Piana RN, Zhao DX, Byrne DW. Association of the metabolic syndrome with pulmonary venous hypertension. Chest 2009; 136: 31-36

23 Cohen AH, Hanson K, Morris K, Fouty B, McMurty IF, Clarke W, Rodman DM. Inhibition of cyclic 3'-5'-guanosine monophosphate-specific phosphodiesterase selectively vasodilates the pulmonary circulation in chronically hypoxic rats. J Clin Invest 1996; 97: 172-179.
24 Cockrill BA, Waxman AB. Phosphodiesterase-5 inhibitors. Handb Exp Pharmacol 2013; 218: 229-255

25 Reffelmann T, Kloner RA. Therapeutic potential of phosphodiesterase 5 inhibition for cardiovascular disease. Circulation 2003; 108: 239-244.

26 Wu X, Yang T, Zhou Q, Li S, Huang L. Additional use of a phosphodiesterase 5 inhibitor in patients with pulmonary hypertension secondary to chronic systolic heart failure: a meta-analysis. Eur J Heart Fail 2014; 16: 444-453.

27 Lewis GD, Lachmann J, Camuso J, Lepore JJ, Shin J, Martinovic ME, Systrom DM, Bloch KD, Semigran MJ. Sildenafil improves exercise hemodynamics and oxygen uptake in patients with systolic heart failure. Circulation 2007; 115: 59-66.

28 Moher D, Liberati A, Tetzlaff J, Altman DG. Preferred reporting items for systematic reviews and meta-analyses: the PRISMA statement. Ann Intern Med 2009; 151: 264-269, W64.

29 Julian PT, Higgins SG. Cochrane Handbook for Systematic Reviews of Interventions (version5.1.0, updated March 2011), 2014.

30 Davis MI, Filion KB, Zhang D, Eisenberg MJ, Afilalo J, Schiffrin EL, Joyal D. Effectiveness of renal denervation therapy for resistant hypertension: a systematic review and meta-analysis. J Am Coll Cardiol 2013; 62: 231-241.

31 Mancini DM, Eisen H, Kussmaul W, Mull R, Edmunds LJ, Wilson JR. Value of peak exercise oxygen consumption for optimal timing of cardiac transplantation in ambulatory patients with heart failure. Circulation 1991; 83: 778-786.

32 Green P, Lund LH, Mancini D. Comparison of peak exercise oxygen consumption and the Heart Failure Survival Score for predicting prognosis in women versus men. Am J Cardiol 2007; 99: 399-403.

33 de Groote P, Dagorn J, Soudan B, Lamblin N, McFadden E, Bauters C. B-type natriuretic peptide and peak exercise oxygen consumption provide independent information for risk stratification in patients with stable congestive heart failure. J Am Coll Cardiol 2004; 43: 1584-1589.

34 Johnson RJ. Gas exchange efficiency in congestive heart failure II. Circulation 2001; 103: 916-918.

35 Wensel R, Georgiadou P, Francis DP, Bayne S, Scott AC, Genth-Zotz S, Anker SD, Coats AJ, Piepoli MF. Differential contribution of dead space ventilation and low arterial pCO2 to exercise hyperpnea in patients with chronic heart failure secondary to ischemic or idiopathic dilated cardiomyopathy. Am J Cardiol 2004; 93: 318-323.

36 Senzaki H, Smith CJ, Juang GJ, Isoda T, Mayer SP, Ohler A, Paolocci N, Tomaselli GF, Hare JM, Kass DA. Cardiac phosphodiesterase 5 (cGMP-specific) modulates betaadrenergic signaling in vivo and is down-regulated in heart failure. FASEB J 2001; 15: 1718-1726.

37 Lepore JJ, Maroo A, Bigatello LM, Dec GW, Zapol WM, Bloch KD, Semigran MJ. Hemodynamic effects of sildenafil in patients with congestive heart failure and pulmonary hypertension: combined administration with inhaled nitric oxide. Chest 2005; 127: 1647-1653.

38 Zhuang $\mathrm{Y}$, Jiang B, Gao H, Zhao W. Randomized study of adding tadalafil to existing ambrisentan in pulmonary arterial hypertension. Hypertens Res 2014; 37 : 507-512.

39 Westerhof N, O'Rourke MF. Haemodynamic basis for the development of left ventricular failure in systolic hypertension and for its logical therapy. J Hypertens 1995; 13: 943-952.

40 Cooper TJ, Guazzi M, Al-Mohammad A, Amir O, Bengal T, Cleland JG, Dickstein K. Sildenafil in Heart failure (SilHF). An investigator-initiated multinational randomized controlled clinical trial: rationale and design. Eur J Heart Fail 2013; 15: 119-122.

Supplementary Information accompanies the paper on Hypertension Research website (http://www.nature.com/hr) 